\title{
Global Games with Noisy Sharing of Information
}

\author{
Behrouz Touri and Jeff Shamma
}

\begin{abstract}
We provide a framework for the study of global games with noisy sharing of information. In contrast to the previous works where it is shown that an intuitive threshold policy is an equilibrium for such games, we show that noisy sharing of information leads to non-existence of such an equilibrium. We also investigate the group best-response dynamics of two groups of agents sharing the same information to threshold policies based on each group's observation and show the convergence of such dynamics.
\end{abstract}

\section{INTRODUCTION}

Global games are examples of games with incomplete information that were used to model pricing debt, currency crises, and mathematical modeling of political opposition [5], [6], [3], [4]. In all these applications, each agent observes an economical or social fundamental through a noisy channel and their decision affect the payoff of all players.

Global games were originally introduced in [1] where two agents observe noisy variants of an underlying signal $\theta$ and they decide to take a binary action. Both the unknown parameter $\theta$ and the decision of the other player influence the payoff of each agent. Therefore, the decision of each player depends on what the other player think she will do which depends what each player thinks the other player thinks what she thinks, and so on so forth. Therefore, such a decision making scenario can be arbitrarily complex and intricate. Since the original work of [1], many works have studied global games and their applications in the study of socio-economical behaviors [5], [6], [3], [4]. The survey paper [7] contains an extensive overview of the results established in this regard. Recently, [2] proposed and studied global games with perfect sharing of information among agents.

\footnotetext{
This work is supported in part by ARO-MURI W911NF-12-10509.

Behrouz Touri is with the Department of Electrical, Computer, and Energy Engineering, University of Colorado, Boulder, email: behrouz.touri@coloradu.edu. Jeff Shamma is with the School of Electrical and Computer Engineering, Georgia Institute of Technology and King Abdullah University of Science and Technology (KAUST), email: shamma@gatech.edu, jeff.shamma@kaust.edu.sa.
}

An interesting feature of many variants of the global games is that often there exists an intuitive equilibrium for such games. By an intuitive equilibrium, we mean a threshold policy based on the expected value of the underlying signal (fundamental) given each agent's observations. This work is an attempt to understand global games under more complex information structure and existence of intuitive equilibrium as well as robustness of the existing results in terms of information available to each agent. We indeed show that such results are not robust to sharing of information: if an agent shares her information, through a noisy channel, no intuitive equilibrium will exist. We also investigate the case of perfect sharing of information among two groups of agents and study the group best-response dynamics for those games.

The structure of this paper is as follows: in Section II, we review the basic setting of global games and in Section III, we propose the extension of this setting under arbitrary information structure. In Section IV, we prove non-existence of linear threshold equilibria for global games with noisy sharing of information and in particular, an intuitive equilibrium. Then, we study global games between two groups of agents and group best-response dynamics in the simple case that agents within each group share the same observation and show the convergence of such best-response dynamics to a unique threshold policy. We conclude our discussion in Section VI.

Notation: We denote the set of real numbers by $\mathbb{R}$. We use $1_{S}$ for the indicator function on a set $S$, i.e. $1_{S}(\alpha)=1$ if $\alpha \in S$ and $1_{S}(\alpha)=0$ for $\alpha \notin S$. We denote the Gaussian distribution with mean $\mu$ and variance $\sigma^{2}$ by $\mathcal{N}\left(\mu, \sigma^{2}\right)$.

\section{Global Games}

In this section, we present the framework of our study. In the simple case of global games, we have $n$ agents and each of the agents decide to either take the risky action $\left(a_{i}=1\right)$ or the safe action $\left(a_{i}=0\right)$. The payoff of an agent taking the safe action is zero and the payoff of an agent taking the risky action is $\sum_{i=1}^{n} a_{i}-\theta$ for some $\theta$. One can think of $\theta$ as 
the strength of a political regime and $\sum_{i=1}^{n} a_{i}$ as the number of people taking the risky action against the regime. One challenging aspect of analyzing global games is that the agents do not observe $\theta$ directly and they observe a noisy version of $\theta$. In that regard, a standard assumption is that agent $i \in[n]$ observes $x_{i}=\theta+\xi_{i}$ where $\left\{\xi_{1}, \ldots, \xi_{n}\right\}$ are independently and identically distributed $\mathcal{N}\left(0, \sigma^{2}\right)$ Gaussian random variables and $\theta$ has some distribution. Although it is rather a non-standard assumption, a common assumption in this context is that $\theta$ is uniformly distributed over $\mathbb{R}$. See [7] and the references therein for discussions on that. It is shown that for the case of $\theta$ with the uniform prior, there is a symmetric threshold policy on $x_{i}$ s which is a Bayesian Nash equilibrium for these games. In other words, there exists a threshold value $t \in \mathbb{R}$ such that for $x_{i} \leq t$, agent $i$ chooses to the take risky action $\left(a_{i}=1\right)$ and for $x_{i}>t$, she takes the safe action and such an action profile leads to an equilibrium. Such a result is intuitively clear: the higher the value $x_{i}$ is, the higher value might $\theta$ have and hence, the riskier taking the risky action is. Here, an important fact is that $x_{i}=\mathrm{E}\left[\theta \mid x_{i}\right]$ which means that in such equilibrium each agent should compare her expected strength of regime given her observation to a threshold and take a proper action accordingly. Such an intuitive result is extended to other other cases of global games. For example, it is shown that if $\theta$ is a $\mathcal{N}\left(y, \tau^{2}\right)$ Gaussian and $y$ is the public knowledge, still there is an equilibrium with threshold policy on $\mathrm{E}\left[\theta \mid x_{i}\right]$ for such games [7].

\section{GlobAl GAMES WITH INFORMATION STRUCTURE}

This work is motivated by understanding the role of information sharing in global games and the structure of emerging equilibria. Here, we discuss the setting under which we study global games with arbitrary information available to each agent. Let $(\Omega, \mathcal{F}, P)$ be a probability space. Let $\theta: \Omega \rightarrow \mathbb{R}$ be a measurable function, perhaps with an improper uniform distribution on $\mathbb{R}$ (i.e. unsigned measure with infinite mass). We assume that agent $i$ has access to some signals related to $\theta$ which is represented by a random vector $\mathbf{s}_{i}: \Omega \rightarrow \mathbb{R}^{d_{i}}$, where $d_{i}$ is a positive integer. We refer to $\mathbf{s}_{i}$ as the private information or simply information of agent $i^{1}$. We refer to a measurable

\footnotetext{
${ }^{1} \mathrm{~A}$ more general formulation of global games with information structure is to assume that agent $i$ s information belongs to a $\sigma$ algebra $\mathcal{F}_{i} \subseteq \mathcal{F}$. Our formulation here is perhaps more intuitive and serves well for the purpose of this study.
}

function $A_{i}: \mathbb{R}^{d_{i}} \rightarrow\{0,1\}$ which maps agent $i$ s private information to an action as a strategy. For any $\omega \in \Omega$, and a given strategy profile $\mathbf{A}=\left(A_{1}, \ldots, A_{n}\right)$ of the $n$ players, let the payoff of agent $i$ be:

$$
u_{i}(\mathbf{A})(\omega)=\left(\sum_{j=1}^{n} A_{j}\left(\mathbf{s}_{j}(\omega)\right)-\theta(\omega)\right) A_{i}\left(\mathbf{s}_{i}(\omega)\right) .
$$

Note that for an outcome $\omega, \sum_{j=1}^{n} A_{j}\left(\mathbf{s}_{j}(\omega)\right)$ is the number of agents taking the risky action, an agent taking the safe action $\left(A_{i}\left(\mathbf{s}_{i}(\omega)\right)=0\right)$ receives payoff zero, and an agent taking the risky action receives the payoff $A_{j}\left(\mathbf{s}_{j}(\omega)\right)-\theta(\omega)$.

\section{A. Structure of an Equilibrium}

In the subsequent discussions, we are interested in the Bayesian Nash equilibria of global games with an information structure. To introduce this concept, let $\mathbf{A}=\left(A_{1}, \ldots, A_{n}\right)$ be the strategy of $n$ agents and let $\mathbf{A}^{-i}$ be the vector which contains the strategies of all agents except the $i$ th agent's strategy. The bestresponse of agent $i$ to the strategy $\mathbf{A}^{-i}$ of other players conditioned on her information $\mathbf{s}_{i}$ is:

$$
B R\left(\mathbf{A}^{-i}\right)= \begin{cases}1 & \text { if } 1+\mathrm{E}\left[\sum_{j \neq i}^{n} A_{j} \mid \mathbf{s}_{i}\right] \geq \mathrm{E}\left[\theta \mid \mathbf{s}_{i}\right] \\ 0 & \text { if } 1+\mathrm{E}\left[\sum_{j \neq i}^{n} A_{j} \mid \mathbf{s}_{i}\right]<\mathrm{E}\left[\theta \mid \mathbf{s}_{i}\right]\end{cases}
$$

We say that $\mathbf{A}=\left(A_{1}, \ldots, A_{n}\right)$ is a Bayesian Nash equilibrium, or simply an equilibrium, if $A_{i}=$ $B R\left(A^{-i}\right)$ for $i=1, \ldots, n$. Throughout the present work, many of the properties and characterizations, such as the equilibrium characterization in Eq. (1) can hold "almost surely" (or with probability one) but for the sake of simplicity and conciseness, we avoid using the term almost surely throughout the following discussion.

The main purpose of this study is to investigate the structure of equilibrium in global games, especially existence of a threshold policy equilibrium, i.e. an equilibrium of the form $\left(1_{f_{1}\left(\mathbf{s}_{1}\right) \leq t_{1}}, \ldots, 1_{f_{n}\left(\mathbf{s}_{n}\right) \leq t_{n}}\right)$ for functions $f_{i}: \mathbb{R}^{d_{i}} \rightarrow \mathbb{R}$ and threshold values $t_{1}, \ldots, t_{n} \in \mathbb{R}$. We say that a threshold equilibrium is linear if $f_{i}: \mathbb{R}^{d_{i}} \rightarrow \mathbb{R}$ is a linear functional for all $i \in$ $\{1, \ldots, n\}$. The intuitive meaning of a threshold policy is clear: each agent evaluates the simple function $f_{i}$ of her observations and compares it to a threshold value if it is less than a threshold, she takes the risky action, and if it is above a threshold value, she takes the safe action. 


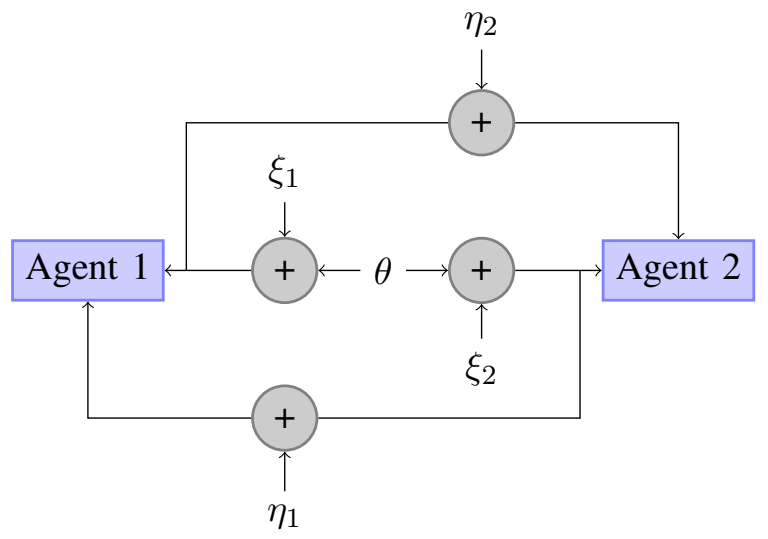

Fig. 1. Global Games with Noisy Sharing of Information

\section{NON-EXISTENCE OF THRESHOLD POLICIES FOR} CORRELATED SIGNALS

As discussed in Section II, for several cases of global games, there exists a threshold equilibrium based on individual agent's expected value of $\theta$ given her private information. In this section, we show that such an intuitive strategy does not lead to an equilibrium if agents share their information.

Let us discuss the setting and the structure of information sharing for this game. Suppose that we have two agents and assume that $\theta$ has a uniform distribution over $\mathbb{R}$. Suppose that agent $i$ observes $x_{i}=\theta+\xi_{i}$ for $i=1,2$, where $\xi_{1}, \xi_{2}$ are independent $\mathcal{N}\left(0, \sigma^{2}\right)$ Gaussian random variables. In addition, suppose that agent 1 also observes a noisy variation of agent 2's private information $x_{2}$, i.e. $y_{1}=x_{2}+\eta_{1}$ where $\eta_{1}$ is a $\mathcal{N}\left(0, \tau^{2}\right)$ Gaussian random variable and similarly, agent 2 also receives $y_{2}=x_{1}+\eta_{2}$ with $\eta_{2}$ being a $\mathcal{N}\left(0, \tau^{2}\right)$ Gaussian random variable. We assume $\theta, \xi_{1}, \xi_{2}, \eta_{1}, \eta_{2}$ are independent. See Fig. 1 for illustration of the information available to each agent. In this case, the information (private signal) of agent $i$ is the vector $\left(x_{i}, y_{i}\right)$. For simplicity, we refer to this game as global games with noisy sharing of information. Let $\bar{\theta}(x, y)=\mathrm{E}\left[\theta \mid x_{i}=x, y_{i}=y\right]$ be the expected value of $\theta$ given agent $i$ s observation $(x, y)$.

The main result of this section is that there is no threshold-policy equilibrium based on $\mathrm{E}[\theta \mid x, y]=$ $\bar{\theta}(x, y)$.

Theorem 1: For $\tau^{2} \in(0, \infty)$, there is no equilibrium with threshold policy on the expected value of $\theta$ given each agent's information, i.e. there do not exist threshold values $t_{1}, t_{2} \in \mathbb{R}$ such that

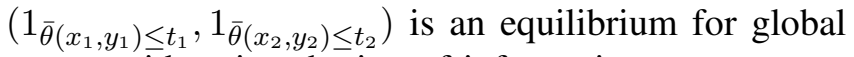
games with noisy sharing of information.
To prove this, we make use of the following properties which follow from the properties of multivariate Gaussian random vectors and their conditional expectations (see Appendix for the proof).

Lemma 1: Let $\theta, x_{1}, x_{2}, y_{1}, y_{2}$ be as defined above and let $\bar{\theta}(x, y)=\frac{\left(\sigma^{2}+\tau^{2}\right) x+\sigma^{2} y}{2 \sigma^{2}+\tau^{2}}$. Then,

a. We have

$$
\bar{\theta}(x, y)=\mathrm{E}\left[\theta \mid x_{1}=x, y_{1}=y\right] .
$$

b. $\left(x_{2}, y_{2}\right)$ conditioned on $\left(x_{1}=x, y_{1}=y\right)$ is a Gaussian random vector with mean:

$$
\mu=\left(\begin{array}{c}
\bar{\theta}(x, y)+\frac{\sigma^{2}(y-\bar{\theta}(x, y))}{\sigma^{2}+\tau^{2}} \\
x
\end{array}\right)
$$

and covariance

$$
\Sigma^{2}=\left(\begin{array}{cc}
\frac{\sigma^{2} \tau^{2}}{\sigma^{2}+\tau^{2}} & 0 \\
0 & \tau^{2}
\end{array}\right)
$$

c. $\theta\left(x_{2}, y_{2}\right)$ conditioned on $\left(x_{1}=x, y_{1}=y\right)$ is a Gaussian random variable with mean

$$
\begin{aligned}
& \mathrm{E}\left[\bar{\theta}\left(x_{2}, y_{2}\right) \mid x_{1}=x, y_{1}=y\right] \\
& \quad=\frac{\tau^{2}}{2 \sigma^{2}+\tau^{2}} \bar{\theta}(x, y)+\frac{\sigma^{2}}{2 \sigma^{2}+\tau^{2}}(x+y) .(2)
\end{aligned}
$$

Using the above properties we can prove Theorem 1: Proof: Suppose that $\left(1_{\bar{\theta}}\left(x_{1}, y_{1}\right) \leq t_{1}, 1_{\bar{\theta}\left(x_{2}, y_{2}\right) \leq t_{2}}\right)$ is an equilibrium for some $t_{1}, t_{2} \in \mathbb{R}$. By Lemma 1-c, $\bar{\theta}\left(x_{2}, y_{2}\right)$ is a Gaussian random variable with mean (2). Therefore, one can find $x, y \in \mathbb{R}$ such that $\bar{\theta}(x, y) \leq$ $t_{1}$ and $\mathrm{E}\left[\bar{\theta}\left(x_{2}, y_{2}\right) \mid x_{1}=x, y_{1}=y\right]$ is an arbitrarily large value. But the variance of $\theta\left(x_{2}, y_{2}\right)$ given $x_{1}, y_{1}$ is a constant function of $\sigma^{2}$ and $\tau^{2}$. Therefore, using Chebyshev's inequality, for the given threshold values $t_{1}, t_{2}$ and an arbitrary $\epsilon>0$, one can find $x, y$ such that $\bar{\theta}(x, y) \leq t_{1}$ and

$$
P\left(\theta\left(x_{2}, y_{2}\right) \leq t_{2} \mid x_{1}=x, y_{1}=y\right) \leq \epsilon .
$$

But by the structure of equilibrium (1), we should have

$$
\begin{aligned}
\epsilon+1 & \geq P\left(\theta\left(x_{2}, y_{2}\right) \leq t_{2} \mid x_{1}=x, y_{1}=y\right)+1 \\
& \geq t_{1}=\bar{\theta}(x, y)=\mathrm{E}\left[\theta \mid x_{1}=x, y_{1}=y\right] .
\end{aligned}
$$

But this inequality holds for for any $\epsilon>0$ and hence, $t_{1} \leq 1$. Note that for any $t_{1}, t$, the set

$$
\begin{aligned}
& E_{t_{1}, t}=\left\{(x, y) \mid \bar{\theta}(x, y) \leq t_{1},\right. \\
& \left.\quad \mathrm{E}\left[\bar{\theta}\left(x_{2}, y_{2}\right) \mid x_{1}=x, y_{1}=y\right]-\bar{\theta}(x, y) \geq t\right\},
\end{aligned}
$$

has a non-zero Lebesgue measure and hence, $P\left(\left(x_{1}, y_{1}\right) \in E_{t_{1}, t}\right)>0$. 
On the other hand, using the same argument for any $\epsilon>0$, one can find $x, y$ such that $\bar{\theta}(x, y)=t_{1}+1>t_{1}$ and

$$
\mathrm{E}\left[\bar{\theta}\left(x_{2}, y_{2}\right) \mid x_{1}=x, y_{1}=x\right],
$$

is arbitrary small and hence,

$$
\begin{aligned}
t_{1}+\epsilon & \geq \bar{\theta}\left(x_{1}, y_{1}\right) \\
& >P\left(\theta\left(x_{2}, y_{2}\right) \leq s_{2} \mid x_{1}=x, y_{1}=y\right)+1 \\
& \geq 1+(1-\epsilon)=2-\epsilon,
\end{aligned}
$$

for any $t_{2} \in \mathbb{R}$. Since, this holds for any $\epsilon>0$, it follows that $t_{1} \geq 2$ which contradicts $t_{1} \leq 1$. Therefore, such a threshold equilibrium does not exist.

Note that $\bar{\theta}(x, y)$ is a linear function of $x, y$. In fact, using Theorem 1, we can generalize this result and show that no linear threshold equilibrium exists for those games.

Theorem 2: Global games with noisy sharing of information do not admit any linear threshold equilibrium.

Proof: Suppose that a global game with noisy sharing of information admits a linear threshold equilibrium $L_{1}\left(x_{1}, y_{1}\right)=\alpha_{1} x_{1}+\beta_{1} y_{1}$ and $L_{2}\left(x_{2}, y_{2}\right)=$ $\alpha_{2} x_{2}+\beta_{2} y_{2}$ with the threshold values $s_{1}, s_{2} \in \mathbb{R}$, respectively. Based on the characterization of an equilibrium in global games with information structure (Eq. (1)), for any $x, y$ such that $L_{1}(x, y) \leq s_{1}$, we should have:

$$
P\left(L_{2}\left(x_{2}, y_{2}\right) \leq t_{2} \mid x, y\right) \geq \bar{\theta}(x, y)-1 .
$$

This implies that $L_{1}(x, y)=\lambda \bar{\theta}(x, y)$ for some $\lambda \in$ $\mathbb{R}$, otherwise, we can find scalars $x, y \in \mathbb{R}$ such that $L_{1}(x, y) \leq t_{1}$ but $\bar{\theta}\left(x_{1}, y_{1}\right)-1 \geq 2$ which contradicts (3). Similarity, it follows that $L_{2}(x, y)=\lambda_{2} \bar{\theta}(x, y)$ for some $\lambda_{2} \in \mathbb{R}$. But any threshold policy on $\lambda \bar{\theta}$ translates to a threshold policy on $\bar{\theta}$ and by Theorem 1 , such a threshold equilibrium does not exist.

\section{Global Games Between Groups of Agents}

Suppose that we have two separated groups of agents $S_{1}, S_{2}$. Agents in the same group, observe the same signal. In this section, we investigate the structure of threshold policies for this information structure. First, we show that at equilibrium, agents belonging to one group should follow the same strategy. In fact, we show a more general result: in global games with arbitrary information sharing scheme, if two agents have the same information, they should follow the same strategy at an equilibrium. Based on this, we study the group best-response dynamics of each group to the other group's threshold policy.

\section{A. Agents with Same Information}

One natural question in the context of global games with information structure is whether same information leads to different strategies and actions or equivalently, can we have contradicting decisions between two agents with similar information in this setting? The answer is negative as shown below.

Lemma 2: Let $\mathbf{s}_{i}=\mathbf{s}_{i^{\prime}}$ for some $i \neq i^{\prime}$ and assume that the strategy profile $\mathbf{A}$ is an equilibrium for a global game with information structure (as described in Section II). Then $A_{i}=A_{i^{\prime}}$.

Proof: Suppose that on the set $\mathcal{E}$, we have $A_{i}\left(\mathbf{s}_{i}(\omega)\right)=1$. Then, the other agent's action on this event is definitely less than or equal to 1, i.e. $A_{i^{\prime}}\left(\mathbf{s}_{i^{\prime}}=\right.$ $A_{i^{\prime}}\left(\mathbf{s}_{i}(\omega)\right) \leq A_{i}\left(\mathbf{s}_{i}(\omega)\right)=1$ on $\mathcal{E}$. Therefore, using (1), on the set $\mathcal{E}$, we have

$$
\begin{aligned}
\mathrm{E}\left[\theta \mid \mathbf{s}_{i^{\prime}}\right] & =\mathrm{E}\left[\theta \mid \mathbf{s}_{i}\right] \leq 1+\mathrm{E}\left[\sum_{j \neq i}^{n} A_{j} \mid \mathbf{s}_{i}\right] \\
& =1+A_{i^{\prime}}\left(\mathbf{s}_{i}^{\prime}\right)+\mathrm{E}\left[\sum_{j \neq i, i^{\prime}}^{n} A_{j} \mid \mathbf{s}_{i}\right] \\
& \leq 1+A_{i}\left(\mathbf{s}_{i}\right)+\mathrm{E}\left[\sum_{j \neq i, i^{\prime}}^{n} A_{j} \mid \mathbf{s}_{i^{\prime}}\right] \\
& =1+\mathrm{E}\left[\sum_{j \neq i^{\prime}}^{n} A_{j} \mid \mathbf{s}_{i}^{\prime}\right] .
\end{aligned}
$$

And hence, $A_{i^{\prime}}\left(\mathbf{s}_{i^{\prime}}\right)=1$. The same argument holds for the event $\mathcal{E}^{\prime}$ where $A_{i^{\prime}}\left(\mathbf{s}_{i^{\prime}}\right)=1$ and hence, $A_{i}\left(\mathbf{s}_{i}\right)=$ $A_{i^{\prime}}\left(\mathbf{s}_{i^{\prime}}\right)$.

\section{B. Equilibrium Structure in Groups}

Consider two groups $S_{1}=\{1, \ldots, m\}$ and $S_{2}=$ $\{m+1, \ldots, m+n\}$ of $m$ and $n$ agents, respectively, such that every agent in the same group observes the same signal. More precisely, let $x_{\ell}=\theta+\xi_{\ell}$ for $\ell=1,2$, where $\theta$ is uniformly distributed on $\mathbb{R}$ and $\xi_{1}, \xi_{2}$ are independent $\mathcal{N}\left(0, \sigma^{2}\right)$ Gaussian random variables. We assume that $\mathbf{s}_{i}=x_{\ell}$ for all $i \in S_{\ell}$ and $\ell=1,2$. As a result of Lemma 2 , in an equilibrium, the agents in the same group should share the same strategy. Motivated by this, in the following discussion, we investigate the group best-response dynamics to the other group's threshold policy. In other words, we study the question of what happens if agents in the same group follow the collective best-response to the agents in the other group assuming that all the agents 
would commit to such a policy. More precisely, let

$$
\left(\left[A_{1}\right]_{m},\left[A_{2}\right]_{n}\right)=(\underbrace{A_{1}, \ldots, A_{1}}_{m \text { times }}, \underbrace{A_{2}, \ldots, A_{2}}_{n \text { times }}) .
$$

We say that $\left(A_{1}^{\prime}, A_{2}^{\prime}\right)$ is a group best-response to $\left(A_{1}, A_{2}\right)$ if $A_{1}^{\prime}=B R\left(\left(\left[A_{1}^{\prime}\right]_{m},\left[A_{2}\right]_{n}\right)^{-i}\right)$ for all $i \in$ $\{1, \ldots, m\}$ and $A_{2}^{\prime}=B R\left(\left(\left[A_{1}\right]_{m},\left[A_{2}^{\prime}\right]_{n}\right)^{-j}\right)$ for all $j \in\{m+1, \ldots, n+m\}$. In this case, $A_{1}^{\prime}$ is the strategy that if all the agents in $S_{1}$ follow it, it would be the best-response to the agents in the other group when all of them are using $A_{2}$. Based on this, we say that $\left\{\left(A_{1}[k], A_{2}[k]\right)\right\}$ is the group best-response dynamics if $\left(A_{1}[k], A_{2}[k]\right)$ is the group best-response to $\left(A_{1}[k-1], A_{2}[k-1]\right)$ for $k>0$.

Theorem 3: Let $A_{1}[0]=1_{x_{1} \leq t_{1}[0]}$ and $A_{2}[0]=$ $1_{x_{2} \leq t_{2}[0]}$ for some $t_{1}[0], t_{2}[0] \in \mathbb{R}$. Then for the group best-response dynamics $\left\{\left(A_{1}[k], A_{2}[k]\right)\right\}$ :

a. There exists a unique threshold $t_{\ell}[k]$ for $\ell=1,2$ and $k>0$ such that $A_{\ell}[k]=1_{x_{\ell} \leq t_{\ell}[k]}$ and also

$$
\begin{aligned}
& n \Phi\left(\frac{t_{2}[k-1]-t_{1}[k]}{\sqrt{2} \sigma}\right)+m=t_{1}[k] \\
& m \Phi\left(\frac{t_{1}[k-1]-t_{2}[k]}{\sqrt{2} \sigma}\right)+n=t_{2}[k],
\end{aligned}
$$

where $\Phi$ is the cumulative distribution function of the normal distribution.

b. Eq. (4) has a unique fixed point $t_{1}^{*}, t_{2}^{*}$ satisfying

$$
\begin{aligned}
(m+n) \Phi\left(\frac{t_{2}^{*}-t_{1}^{*}}{\sqrt{2} \sigma}\right) & =n-\left(t_{2}^{*}-t_{1}^{*}\right) \\
t_{1}^{*} & =n \Phi\left(\frac{t_{2}^{*}-t_{1}^{*}}{\sqrt{2} \sigma}\right)+m .
\end{aligned}
$$

c. For $\ell=1,2$ and $k \geq 2$, we have

$$
\left|t_{\ell}[k]-t_{\ell}^{*}\right| \leq \frac{1}{\left(1+\frac{2 \sqrt{\pi} \sigma}{m}\right)\left(1+\frac{2 \sqrt{\pi} \sigma}{n}\right)}\left|t_{\ell}[k-2]-t_{\ell}^{*}\right|,
$$

and hence, $t_{\ell}[k]$ converges to $t_{\ell}^{*}$.

Proof:

a. Suppose that the strategy of all agents $i=1, \ldots, m$ in $S_{1}$ is $1_{x_{1} \leq t_{1}}$. Then, if agents in $S_{2}$ observe $x_{2}=$ $x$, the payoff of the risky action would be:

$$
\begin{gathered}
m P\left(x_{1} \leq t_{1} \mid x_{2}=x\right)+n-\mathrm{E}\left[\theta \mid x_{2}=x\right] \\
=m P\left(x_{1} \leq t_{1} \mid x_{2}=x\right)+n-x .
\end{gathered}
$$

But $x_{1}$ conditioned on $x_{2}=x$ is a Gaussian with mean $x$ and variance $2 \sigma^{2}$. Therefore, agents in $S_{2}$ decide to take risky action if $m \Phi\left(\frac{t_{1}-x}{\sqrt{2} \sigma}\right)+n-x \geq$ 0 . The function $m \Phi\left(\frac{t_{1}-x}{\sqrt{2} \sigma}\right)+n-x$ is a strictly decreasing function of $\beta$ (ranging from $+\infty$ to $-\infty)$ and hence, has a unique root $\beta\left(t_{1}\right)$ satisfying:

$$
m \Phi\left(\frac{t_{1}-\beta\left(t_{1}\right)}{\sqrt{2} \sigma}\right)+n=\beta\left(t_{1}\right) .
$$

As a result, for $x_{2} \leq \beta\left(t_{1}\right)$ taking the risky action is beneficial to agents in $S_{2}$ and for $x_{2}>\beta\left(t_{1}\right)$ taking risky action is not beneficial. Similarly, for the group best-response to the strategy $1_{x_{2} \leq t_{2}}$, the bestresponse is the unique strategy $1_{x_{1} \leq \alpha\left(t_{2}\right)}$ satisfying:

$$
n \Phi\left(\frac{t_{2}-\alpha\left(t_{2}\right)}{\sqrt{2} \sigma}\right)+m=\alpha\left(t_{2}\right)
$$

b. Let $d$ be the unique solution of the following equation (which exists and is unique because of monotonicity):

$$
(m+n) \Phi\left(\frac{d}{\sqrt{2} \sigma}\right)=n-d .
$$

It is not hard to check that $\left(t_{1}^{*}, t_{2}^{*}\right)$ defined by $t_{1}^{*}=$ $n \Phi\left(\frac{d}{\sqrt{2} \sigma}\right)+m$ and $t_{2}^{*}=d+t_{1}^{*}$ is a fixed point of (4). On the other hand, subtracting (6) and (5) implies that for any fixed point $\left(t_{1}^{*}, t_{2}^{*}\right), d=t_{2}^{*}-t_{1}^{*}$ should satisfy (7) and hence, such a fixed point is unique.

c. Let $\mu(s)=\frac{1}{\sqrt{4 \pi \sigma^{2}}} e^{-\frac{s^{2}}{4 \sigma^{2}}}$ be the probability density function of a $\mathcal{N}\left(0,2 \sigma^{2}\right)$ Gaussian random variable. If we subtract both sides of (4) from the corresponding fixed point equations, we have

$$
\begin{aligned}
& n \int_{t_{2}^{*}-t_{1}^{*}}^{t_{2}[k-1]-t_{1}[k]} \mu(s) d s=\left(t_{1}[k]-t_{1}^{*}\right), \\
& m \int_{t_{1}^{*}-t_{2}^{*}}^{t_{1}[k-1]-t_{2}[k]} \mu(s) d s=\left(t_{2}[k]-t_{2}^{*}\right) .
\end{aligned}
$$

By the mean-value theorem for the integrals, for some $\lambda_{1}[k]$ between $t_{2}[k-1]-t_{1}[k]$ and $t_{2}^{*}-t_{1}^{*}$ and some $\lambda_{2}[k]$ between $t_{1}^{*}-t_{2}^{*}$ and $t_{1}[k-1]-t_{2}[k]$, we have:

$$
\begin{aligned}
n\left(\left(t_{2}[k-1]-t_{1}[k]\right)\right. & \left.-\left(t_{2}^{*}-t_{1}^{*}\right)\right) \\
\times \mu\left(\lambda_{1}[k]\right) & =\left(t_{1}[k]-t_{1}^{*}\right) \\
m\left(\left(t_{2}[k]-\right.\right. & \left.\left.t_{1}[k-1]\right)-\left(t_{1}^{*}-t_{2}^{*}\right)\right) \\
\times \mu\left(\lambda_{2}[k]\right) & =\left(t_{2}[k]-t_{2}^{*}\right)
\end{aligned}
$$

and hence,

$$
\begin{aligned}
\left(t_{1}[k]-t_{1}^{*}\right) & =\frac{n \mu\left(\lambda_{1}[k]\right)}{1+n \mu\left(\lambda_{1}[k]\right)}\left(t_{2}[k-1]-t_{2}^{*}\right) \\
\left(t_{2}[k]-t_{2}^{*}\right) & =\frac{m \mu\left(\lambda_{2}[k]\right)}{1+m \mu\left(\lambda_{2}[k]\right)}\left(t_{1}[k-1]-t_{1}^{*}\right) .
\end{aligned}
$$


Using the same equality for the best-response strategies at time $k-1$ and $k-2$, we have:

$$
\begin{aligned}
& \left(t_{1}[k]-t_{1}^{*}\right)= \\
& \frac{n \mu\left(\lambda_{1}[k]\right)}{1+n \mu\left(\lambda_{1}[k]\right)} \frac{m \mu\left(\lambda_{2}[k-1]\right)}{1+m \mu\left(\lambda_{2}[k-1]\right)}\left(t_{1}[k-2]-t_{1}^{*}\right) \\
& \left(t_{2}[k]-t_{2}^{*}\right)= \\
& \frac{m \mu\left(\lambda_{2}[k]\right)}{1+m \mu\left(\lambda_{2}[k]\right)} \frac{n \mu\left(\lambda_{1}[k-1]\right)}{1+n \mu\left(\lambda_{1}[k-1]\right)}\left(t_{2}[k-2]-t_{2}^{*}\right) .
\end{aligned}
$$

As a result, for $\ell=1,2$, we have

$$
\left|t_{\ell}[k]-t_{\ell}^{*}\right| \leq \frac{1}{\left(1+\frac{2 \sqrt{\pi} \sigma}{m}\right)\left(1+\frac{2 \sqrt{\pi} \sigma}{n}\right)}\left|t_{\ell}[k-2]-t_{\ell}^{*}\right|,
$$

and hence, $\lim _{k \rightarrow \infty} t_{\ell}[k]=t_{\ell}^{*}$.

By the characterization of the fixed point $\left(t_{1}^{*}, t_{2}^{*}\right)$ in Theorem 3-b, the larger group has a higher threshold at equilibrium (i.e. if $n>m$, we have $t_{2}^{*}>t_{1}^{*}$ ) which means that for the smaller group, the group bestresponse dynamics converges to a more conservative strategy.

\section{Conclusion And Suggestions FOR FURTHER STUDIES}

In this work, we investigated the role of information sharing in global games. We showed non-existence of threshold policies on the expected value of the economic fundamental conditioned on agents' information when agents share their information (through a noisy channel). We also studied an extreme case of sharing information when agents in two groups play global games and agents in the same group share the same information. We showed the uniqueness of threshold policies in this case and convergence of the group bestresponse dynamics.

For the global games with noisy sharing of information, finding a threshold strategy and how it is shaped by the sharing of information is left as an open problem for future studies.

\section{APPENDIX}

Here, we briefly discuss the derivation of the properties in Lemma 1 . To simplify notation, we use $P(h)$ or $\mathrm{E}[h]$ instead of $P(H=h)$ or $\mathrm{E}[H=h]$ for some random variable (vector) $H$ and a realization $h$. For the conditional probability of $\left(x_{2}, y_{2}\right)$ given $\left(x_{1}, y_{1}\right)$ under uniform distribution assumption for $\theta$, we have:

$$
\begin{aligned}
& P(\theta \mid x, y)=\frac{P(x, y \mid \theta) P(\theta)}{P(x, y)} \\
& =\frac{P(x, y \mid \theta) P(\theta)}{\int_{-\infty}^{\infty} P(x, y \mid \lambda) P(\lambda) d \lambda}=\frac{P(x, y \mid \theta)}{\int_{-\infty}^{\infty} P(x, y \mid \lambda) d \lambda} .
\end{aligned}
$$

But $P(x, y \mid \lambda)=\frac{1}{2 \pi \sqrt{\sigma^{2}\left(\sigma^{2}+\tau^{2}\right)}} e^{-\frac{(x-\lambda)^{2}}{2 \sigma^{2}}} e^{-\frac{(y-\lambda)^{2}}{2\left(\sigma^{2}+\tau^{2}\right)}}$. Using the properties of exponential integrals and exponential integral tables, it follows that:

$$
\int_{-\infty}^{\infty} P(x, y \mid \lambda) d \lambda=\frac{1}{\sqrt{2 \pi\left(2 \sigma^{2}+\tau^{2}\right)}} e^{-\frac{(x-y)^{2}}{2\left(2 \sigma^{2}+\tau^{2}\right)}}
$$

Therefore, $\mathrm{E}[\theta \mid x, y]=\frac{\left(\sigma^{2}+\tau^{2}\right) x+\sigma^{2} y}{2 \sigma^{2}+\tau^{2}}$.

For computing $\mathrm{E}\left[\bar{\theta}\left(x_{2}, y_{2}\right) \mid x_{1}=x, y_{1}=y\right]$, first let us derive the conditional distribution of $x_{2}, y_{2}$ given $x_{1}, y_{1}$. For a given $\theta,\left(x_{1}, y_{1}, x_{2}, y_{2}\right)$ is a Gaussian random vector with mean $\mu^{\prime}$ and covariance matrix $\Sigma^{\prime}$

$\mu^{\prime}=\left(\begin{array}{c}\theta \\ \theta \\ \theta \\ \theta\end{array}\right), \Sigma^{\prime}=\left(\begin{array}{cccc}\sigma^{2} & 0 & 0 & \sigma^{2} \\ 0 & \sigma^{2}+\tau^{2} & \sigma^{2} & 0 \\ 0 & \sigma^{2} & \sigma^{2} & 0 \\ \sigma^{2} & 0 & 0 & \sigma^{2}+\tau^{2}\end{array}\right)$.

Therefore, using the properties of multivariate Gaussian vectors (see e.g. [8]), $x_{2}, y_{2}$ given $x_{1}=x, y_{1}=y$ will be a Gaussian random vector with mean:

$\mu=\left(\begin{array}{c}\bar{\theta}(x, y)+\frac{\sigma^{2}(y-\bar{\theta}(x, y))}{\sigma^{2}+\tau^{2}} \\ x\end{array}\right), \sigma=\left(\begin{array}{cc}\frac{\sigma^{2} \tau^{2}}{\sigma^{2}+\tau^{2}} & 0 \\ 0 & \tau^{2}\end{array}\right)$.

As a result, we have:

$$
\begin{aligned}
& \mathrm{E}\left[\theta\left(x_{2}, y_{2}\right) \mid x_{1}=x, y_{1}=y\right] \\
& =\mathrm{E}\left[\frac{\left(\sigma^{2}+\tau^{2}\right) x_{2}+\sigma^{2} y_{2}}{2 \sigma^{2}+\tau^{2}} \mid x, y\right] \\
& =\frac{\tau^{2}}{2 \sigma^{2}+\tau^{2}} \bar{\theta}(x, y)+\frac{\sigma^{2}}{2 \sigma^{2}+\tau^{2}}(x+y) .
\end{aligned}
$$

\section{REFERENCES}

[1] H. Carlsson and E. van Damme, Global games and equilibrium selection, Econometrica 61 (1993), 989-1018.

[2] M. Dahleh, A. Tahbaz-Salehiz, J.N. Tsitsiklis, and S. Zoumpoulis, Coordination with local information, July 2013.

[3] I. Goldstein, Interdependent banking and currency crises in a model of self- fulfilling beliefs, Ph.D. thesis, 2000, University of Tel Aviv.

[4] S. Morris, Contagion, Review of Economic Studies 67 (2000), $57-78$.

[5] S. Morris and H. S. Shin, Unique equilibrium in a model of self-fulfilling currency attacks, American Economic Review $\mathbf{8 8}$ (1998), 587=597.

[6] S. Morris and H. S. Shin, Coordination risk and the price of debt, (1999b), Available at http://www.econ.yale.edu/smorris.

[7] , Global games: theory and applications, Econometric Society Monographs 35 (2003), 56-114.

[8] C.E. Rasmussen and C.K.I. Williams, Gaussian processes for machine learning, Adaptative computation and machine learning series, University Press Group Limited, 2006. 